\title{
MODELLING AND SIMULATION OF FILTER ADAPTATION BY DAPHNIA
}

\author{
Tibor Kmet \\ Department of Informatics, Faculty of Natural Sciences \\ Constantine the Philosopher University, Tr. A. Hlinku 1 \\ 94974 Nitra Slovak Republic \\ Email: tkmet@ukf.sk \\ Maria Kmetova \\ Department of Mathematics, Faculty of Natural Sciences \\ Constantine the Philosopher University, Tr. A. Hlinku 1 \\ 94974 Nitra Slovak Republic \\ Email: mkmetova@ukf.sk
}

\section{KEYWORDS}

mathematical model of ecosystem, stability analysis, feeding adaptation of Daphnia, optimal control problem, adaptive critic and recurrent neural network, numerical simulation

\section{ABSTRACT}

A model of feeding adaptation of a filter feeder in the framework of a pelagic ecosystem is constructed by modifying the simplified ecosystem model AQUAMOD for the presence of several different sized species of algae. Stability analysis of equilibria and some numerical simulation is given. It is shown that Hopf bifurcation may occur depending on filtration rate. Also the assumption that filtration adaptability represents optimization type process is incorporated. Two possible strategies were followed: an instantaneous optimality at each time interval and an integral formulation, maximization of the integral biomass. The optimal control problem is transcribed into nonlinear programming problem, which is implemented with adaptive critic neural network and recurrent neural network for solving nonlinear projection equations. Results show that adaptive critic based systematic approach and neural network solving of nonlinear equations hold promise for obtaining the optimal control with control and state constraints.
\end{abstract}

\section{MODEL DESCRIPTION}

In this paper we consider a simplified ecosystem model AQUAMOD for the presence of several different sized species of algae. The model consists of phosphorus $\left(x_{1}\right)$ as a limiting nutrient for growth of four species of different sized algae $\left(x_{2}-x_{5}\right)$ and zooplankton $\left(x_{6}\right)$. Similar models of $n$ species of microorganisms competing exploitatively for one, two or more growth-limiting nutrients are used to study continuous culture of microorganisms in chemostat under constant condition (Smith et al., 1995) without any predators. The model is described by the following system of ordinary differential equation (1):

$$
\begin{aligned}
\dot{x}_{1}= & a_{7}\left(a_{8}-x_{1}\right)-\sum_{i=2}^{5}\left(\frac{d_{1} x_{i} p_{i} x_{1}}{x_{1}+s_{i}}\right. \\
& \left.-r_{i} f_{2} x_{i}+x_{i} x_{6} C_{i}\left(1-\frac{d_{4}}{a_{4}+x_{i}}\right)\right), \\
\dot{x}_{i}= & \frac{d_{1} x_{i} p_{i} x_{1}}{x_{1}+s_{i}}-r_{i} f_{2} x_{i}-x_{i} x_{6} E_{i}-d_{2} x_{i}+a_{i+9} a_{7} \\
& \text { for } i=2 \ldots 5, \\
\dot{x}_{6}= & x_{6}\left(d_{3} \sum_{i=2}^{5} \frac{C_{i} x_{i}}{a_{4}+x_{i}}-a_{5}\right)+a_{6} .
\end{aligned}
$$

Functions occuring in the model are given in Tables 2 in ecological and mathematical notation, respectively. For detail explanation see (Kmet et al., 2004). It is derived from the models of the series AQUAMOD (Straskraba et al., 1985) modified by the inclusion of several "species" of algae. Description of the light dependence of algae is highly simplified. Instead of an approximative integration of the algal growth over depth and time distribution of light intensity only a simple function $g(I)$ is used, describing Michaelis-Menten type dependence with the halfsaturation constant for light $I K M$. We consider this oversimplification appropriate for the purposes of this paper.

Four species of algae were considered during the computations performed: $x_{2}, \ldots, x_{5}$. Each "species" is represented by a particular algal cell (or colony) volume. The volumes were set arbitrarily to $\left(V_{i}=50,500,2500\right.$ and $5000 \mu \mathrm{m}^{3}$ ), to approximate the set of "edible" algal sizes commonly occurring in our reservoirs. The ecological parameters of the algae are considered to be the functions of $V_{i}$ ((Straskraba et al., 1985)). Table 1 gives the corresponding values used in the present simulations.

However, for other values of $V_{i}$ it is possible to derive the parameters from the functions $P_{\max }\left(V_{i}\right), K S\left(V_{i}\right)$ and $\operatorname{Resp}\left(V_{i}\right)$ given in Table 3 . It is to be noted that $P_{\text {max }}$ corresponds to light saturation and temperature of $0^{\circ} \mathrm{C}$; for $20^{\circ} \mathrm{C}$ the growth rate will be about 7.2 times higher. The high values of PRFOS are used to sim- 
Table 1: Values of parameters

\begin{tabular}{|c|c|c|}
\hline$a_{1}$ & 0.05 & sedimentationrate $\left[\right.$ day $\left.^{-1}\right]$ \\
\hline$a_{2}$ & 0.6 & $\begin{array}{l}\text { maximumefficiencyof } \\
\text { zooplanktonassimilation }\end{array}$ \\
\hline$a_{3}$ & 0.05 & $\begin{array}{l}\text { recalculation fromunitsofalgae } \\
\text { tounitsof zooplankton }\end{array}$ \\
\hline$a_{4}$ & 60 & $\begin{array}{l}\text { halfsaturationconstantforzooplankton } \\
\text { feeding }\left[\mathrm{mg} \cdot \mathrm{m}^{-3} \mathrm{CHA}\right]\end{array}$ \\
\hline$a_{5}$ & 0.03 & zooplanktonmortality $\left[d^{-1}\right]$ \\
\hline$a_{6}$ & 0.002 & in flowof zooplankton $\left[m^{-3}\right.$ C.day $\left.y^{-1}\right]$ \\
\hline$a_{7}$ & 0.1 & hydraulicloading $\left[d^{-1}\right]$ \\
\hline$a_{8}$ & 200 & inflowphosphorusconcentration $\left[\mathrm{mg} \cdot \mathrm{m}^{-3} \mathrm{P}\right]$ \\
\hline$a_{9}$ & 0.9 & zooplanktonfiltrationrate $\left[m^{-3}\right.$ C.day $\left.{ }^{-1}\right]$ \\
\hline$a_{10}$ & 120 & $\begin{array}{l}\text { halfsaturationconstantforlight } \\
{\left[\text { cal.cm }^{-2} \cdot \text { day }^{-1}\right]}\end{array}$ \\
\hline$a_{11}-a_{14}$ & 0 . & $\begin{array}{l}\text { inflowof phytoplanktonconcentration } \\
{\left[\mathrm{mg} \cdot \mathrm{m}^{-3} \mathrm{CHA}\right]}\end{array}$ \\
\hline
\end{tabular}

ulate eutrophic conditions. For the filtration capability of zooplankton we assume that algal volumes selected at a given setting of the filtratory apparatus have lognormal distribution. This is identical with the "size limited predators"and the function we propose is approximately identical with the "selectivity" by this class of predators as given by (Zaret, 1980).

Table 2: Size-specific parameters of algae

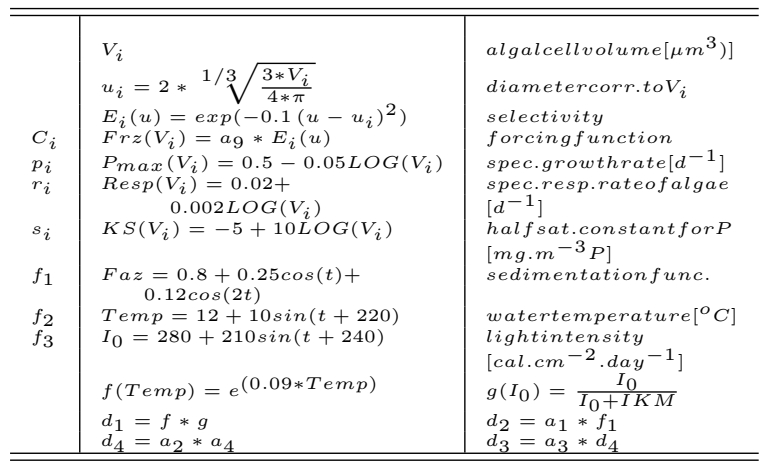

The description of selectivity $E_{i}$ is as follows:

$$
E_{i}(u)=\exp \left(-0.1\left(u-u_{i}\right)^{2}\right)
$$

where $u$ is the value of setal density directly related to the algal diameter for which selectivity is maximal and $u_{i}$ is the diameter corresponding to each algal cell volume $V_{i}$. The specific filtration rate of algae of different sizes (volumes) of the population adapted to certain condition, i.e., with certain values of $u$ becomes

$$
\operatorname{Frz}\left(V_{i}\right)=a_{9} * E_{i}(u),
$$

where $a_{9}$ is the filtration rate for algae of the optimal size, i.e., those which are filtered with the selectivity factors $E_{i}\left(u_{i}\right)=1$.

\section{GLOBAL BEHAVIOR OF THE MODEL}

We assume in this section that $E_{i}(u)=$ const. for $i=2,3,4,5$. We also assume that $a_{j}>0$ for
Table 3: Parameters for four "species" of algea

\begin{tabular}{c|c|c|c|c}
\hline \hline$V_{i}$ & 50 & 500 & 2500 & 5000 \\
$u_{i}$ & 4.57 & 9.85 & 16.84 & 21.22 \\
$p_{i}$ & 0.4151 & 0.3651 & 0.3301 & 0.3151 \\
$s_{i}$ & 11.99 & 21.99 & 28.98 & 31.99 \\
$r_{i}$ & 0.023 & 0.025 & 0.027 & 0.028 \\
\hline \hline
\end{tabular}

$j=5, \ldots, 14$. We will show that under these assumptions and the effect of periodically varying environmental conditions our system has a periodic solution with the one year period (360 days).

Let us denote

$$
z(t)=x_{1}(t)+\ldots+x_{6}(t) .
$$

Denote further

$$
b=\min \left(a_{7}, d_{2}, a_{5}\right), a=a_{7}\left(a_{6}+a_{11}+\ldots+a_{14}\right) .
$$

It follows that

$$
\dot{z}(t) \leq-z(t) b+a .
$$

By variation of constants we obtain

$$
z(t) \leq \exp (-b t)\left(z_{0}-a\right)+a,
$$

where $z_{0}=x_{10}+\ldots+x_{60}$ and $x_{0}$ is the initial condition of the solution $x\left(t, t_{0}, x_{0}\right)$, i.e. $x\left(t_{0}, t_{0}, x_{0}\right)=$ $x_{0}$.

It follows that

$$
\lim _{t \rightarrow \infty} z(t) \leq a .
$$

Now we will demonstrate that the solutions of the system are ultimately bounded, i.e., there exist $B>0$ and $T>0$ such that for all solutions $x\left(t, t_{0}, x_{0}\right)$ of the system holds that $x\left(t, t_{0}, x_{0}\right)<B$ for all $t>t_{0}+T$, where $B$ does not depend on $t_{0}$ and $x_{0}$ and $T$ can depend on $t_{0}$ and $x_{0}$. Set $T=1$ for $z_{0} \leq a+1$, $T=\left(\ln \left(z_{0}-a\right)\right) / b$ for $z_{0}>a+1$ and $B=3(a+1)$. It evidently holds true that $x\left(t, t_{0}, x_{0}\right)<B$ for $t>$ $t_{0}+T$. The solutions of the system are therefore ultimately bounded. According to (Yoshizawa, 1975) there exists a periodic solution with the period of 360 days and the following proposition is true.

Proposition 1 System (1) under periodically varying environmental conditions with period 360 days has a periodic solution with the same period.

During constant density of setal ( $u=$ const.) the zooplankton captures only phytoplankton of a certain size range dependent on $u$. The growth of other species of algae which are not at all or only to a limited extent consumed is therefore limited by nutrients mainly. We will demonstrate that the species which is not intensively consumed by zooplankton and has therefore the best conditions for development dominates in the struggle for existence over other species. 
Assume further that $a_{i}=0$ for $i=11, . .14$.

Derivatives $\dot{x}_{i}$ for $i=2, \ldots, 5$ can be written in the following way

$$
\dot{x}_{i}=x_{i} F_{i}(x, t)
$$

where

$F_{i}(x, t)=d_{1} p_{i} x_{1} /\left(x_{1}+s_{i}\right)-E_{i} x_{6}-d_{2}-r_{i} f_{2}$.

As follows from the shape of functions $p_{i}$ and $s_{i}$ the following holds true:

$$
p_{i}>p_{j}, r_{i}<r_{j} \text { and } s_{i}<s_{j} \text { for } i<j .
$$

Therefore

$$
p_{i} /\left(x_{1}+s_{i}\right)>p_{j} /\left(x_{1}+s_{j}\right) \text { for } i<j .
$$

In respect to the shape of the function $E_{i}$ for $u$ sufficiently large $\left(u>u_{2}\right)$

$$
E_{2}<E_{j} \text { for } j=3,4,5 .
$$

Calculate the derivatives

$$
\left(\frac{\dot{x_{i}}}{x_{j}}\right)=\frac{x_{i}}{x_{j}}\left(F_{i}(x, t)-F_{j}(x, t)\right) .
$$

In respect to (3) and (4) the following holds true

$$
F_{2}(x, t)>F_{j}(x, t) \text { for } j=3,4,5 .
$$

By variation of constants we obtain

$$
\frac{x_{2}(t)}{x_{j}(t)}=\frac{x_{2}(0)}{x_{j}(0)} \exp \left(\int_{0}^{t}\left(F_{2}(x, s)-F_{j}(x, s)\right) d s\right) .
$$

It is true that

$$
\lim _{t \rightarrow \infty} \frac{x_{2}(t)}{x_{j}(t)}=\infty
$$

Due to the boundedness of the solutions, $x_{j}(t)$ converges to zero for $j=3,4,5$ and the following proposition is true.

Proposition 2 In respect to (2), (3) and u sufficiently large the species of algae are not able to coexist.

Remark In a similar way we can show that for $x_{2}=$ $0 x_{4}$ and $x_{5}$ go extinct for $t \rightarrow \infty$.

\section{STABILITY ANALYSIS OF EQUILIBRIA}

In this section we investigate the effects of an increasing filter density $u$ under constant environmental condition. One parameter analysis of existence and stability of equilibria of (1) is carried out using filter density $u$ as a bifurcation parameter. We consider equilibrium solutions to exist only if they lie in the nonnegative cone. Derivatives $\dot{x}_{i}$ for $i=2, \ldots, 5$ can be written in the following way

$$
\dot{x}_{i}=x_{i} F_{i}(x, u) .
$$

Suppose that $x_{6}=0$ and $a_{i+9}=0, i=2,3,4,5$. Then we have the following 4 equilibrium points: $\hat{x}^{i 0}=\left(\hat{x}_{1 i}, \hat{x}_{2}, \ldots, \hat{x}_{5}, 0\right)$, where

$$
\begin{aligned}
\hat{x}_{1 i}= & \frac{s_{i} *\left(r_{i} * f_{2}+d_{2}\right)}{d_{1} * p_{i}-r_{i} * f_{2}-d_{2}}, \\
\hat{x}_{i}= & \frac{a_{7} *\left(a_{8}-x_{1 i}\right)}{\frac{d 1 * p_{i} * x_{1}}{x_{1}+s_{i}}-r_{i} * f 2}, \\
& \text { for } i=2, \ldots, 5 \\
\hat{x}_{j}= & 0, \text { for } j=2, \ldots, 5, j \neq i .
\end{aligned}
$$

Jacobian matrix at equilibrium point $\hat{x}^{20}$ has negative eigenvalues, i.e., it is locally asymptotically stable. The other $\hat{x}^{i 0}, i=3,4,5$ have at least one positive eigenvalue, and they are unstable. Suppose now that $x_{i}=0$ and $a_{i+9}=0, i=2,3,4,5$. Then we have the following equilibrium point

$$
\hat{x}^{0}=\left(a_{8}, 0,0,0,0, \frac{a_{6}}{a_{5}}\right) .
$$

The eigenvalues of Jacobian matrix $J$ at $\hat{x}^{0}$ are:

$$
\begin{aligned}
\lambda_{1} & =-a_{7} \\
\lambda_{i} & =\frac{d_{1} p_{i} a_{8}}{s_{i}+a_{8}}-r_{i} f_{2}-\frac{a_{6}}{a_{5}} E_{i}-d_{2} \text { for } i=2,3,4,5 \\
\lambda_{6} & =-a_{5} .
\end{aligned}
$$

It follows from the simple calculation that if $a_{8}>$ $\frac{r_{i} f_{2}+d_{2}+a_{6} / a_{5} E_{i}}{d_{1} p_{i}-r_{i} f_{2}-d_{2}-a_{6} / a_{5} E_{i}}>0$, then equilibrium point $\hat{x}^{0}$ is unstable. By similar way as (Hsu et al., 1977) we can show that if $b_{i}=d_{1} p_{i}-r_{i} f_{2}-d_{2}-a_{6} / a_{5} E_{i} \leq 0$ then

$$
\lim _{t \rightarrow \infty} x_{i}(t)=0 \text {. }
$$

Suppose that $b_{i}>0$ and let us consider the existence of "interior" equilibrium points, where $\hat{x}_{i}>0$ for some $i=2,3,4,5$. Define

$$
\begin{aligned}
R_{i}= & \left\{x \in R^{6} \mid x_{1}, x_{i}, x_{6} \geq 0, x_{j}=0,\right. \\
& \text { for } j=2, \ldots, 5, j \neq i\} \text { for } i=2, \ldots, 5 \\
R_{i k}= & \left\{x \in R^{6} \mid x_{1}, x_{i}, x_{k}, x_{6} \geq 0, x_{j}=0,\right. \\
& \text { for } j=2, \ldots, 5, j \neq i, j \neq k\} \\
& \text { for } i=2, \ldots, 5, j=i+1, \ldots, 5
\end{aligned}
$$

Coordinates $\hat{x}_{1}, \hat{x}_{6}$ of equilibrium points are defined by the condition $F_{2}(x, u)=F_{3}(x, u)=F_{4}(x, u)=$ $F_{5}(x, u)=0$. For a given set of parameters and function there are ten types of equlibrium points depending on filtration rate $u$ :

$$
\hat{x}^{1}=\left(\hat{x}_{1}, \hat{x}_{2}, 0,0,0, \hat{x}_{6}\right) \in R_{2}
$$




$$
\begin{aligned}
\hat{x}^{2} & =\left(\hat{x}_{1}, 0, \hat{x}_{3}, 0,0, \hat{x}_{6}\right) \in R_{3} \\
\hat{x}^{3} & =\left(\hat{x}_{1}, 0,0, \hat{x}_{4}, 0, \hat{x}_{6}\right) \in R_{4} \\
\hat{x}^{4} & =\left(\hat{x}_{1}, 0,0,0, \hat{x}_{5}, \hat{x}_{6}\right) \in R_{5} \\
\hat{x}^{5} & =\left(\hat{x}_{1}, \hat{x}_{2}, \hat{x}_{3}, 0,0, \hat{x}_{6}\right) \in R_{23} \\
\hat{x}^{6} & =\left(\hat{x}_{1}, \hat{x}_{2}, 0, \hat{x}_{4}, 0, \hat{x}_{6}\right) \in R_{24} \\
\hat{x}^{7} & =\left(\hat{x}_{1}, \hat{x}_{2}, 0,0, \hat{x}_{5}, \hat{x}_{6}\right) \in R_{25} \\
\hat{x}^{8} & =\left(\hat{x}_{1}, 0, \hat{x}_{3}, \hat{x}_{4}, 0, \hat{x}_{6}\right) \in R_{34} \\
\hat{x}^{9} & =\left(\hat{x}_{1}, 0, \hat{x}_{3}, 0, \hat{x}_{5}, \hat{x}_{6}\right) \in R_{35} \\
\hat{x}^{10} & =\left(\hat{x}_{1}, 0,0, \hat{x}_{4}, \hat{x}_{5}, \hat{x}_{6}\right) \in R_{45}
\end{aligned}
$$

Determining stability of equilibria is accomplished by linearizing the model about steady state and examining the eigenvalues. For the presented model 16 possible kind of equilibria can exist on nonnegative cone. Jacobian matrix $J$ about equilibria $\hat{x}^{5}, \hat{x}^{6}, \hat{x}^{7}, \hat{x}^{8}$ and $\hat{x}^{20}$ for different value of $u$ has eigenvalues with negative real part. The simulations seem to indicate that depending on $u$ solution of (1) converges to one of equilibria $\hat{x}^{5}, \hat{x}^{6}, \hat{x}^{7}, \hat{x}^{8}$ and $\hat{x}^{20}$ or to periodic solution (Fig. 2). Figs. 1, 2 and 3 show the dynamics of algae in a

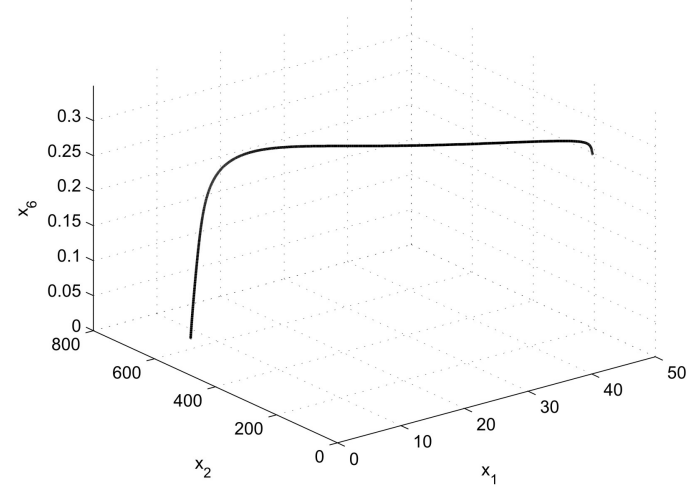

Figure 1: Numerical solution of system (1) for initial condition $x_{1}^{0}=40.2, x_{2}^{0}=0.5, x_{3}^{0}=0.5, x_{4}^{0}=$ $14.2, x_{5}^{0}=497.9, x_{6}^{0}=0.3, u=12$ and constant environmental condition $(t=120$ in Table 1$)$

simplified aquatic ecosystem simulating the presence of zooplankton of different body size and correspondingly different filter density $u$ under constant environmental condition $(\mathrm{t}=120)$. The comparison of three figures for selected arbitrary constant values of $u$ demonstrates that not only the size but also the number of algal species surviving in the system depends on $u$. For the environmental conditions specified in the given simulation experiment and $u=12$ (Fig. 1) the algal sizes $x_{j}$ for $j=3,4,5$ converge to zero and only the smallest phytoplankton species $x_{2}$ survives and the solution converges to $\hat{x}^{20}$, (Proposition 2). When $u$ is set to 1.5 or 10.5 two species of algae are able to coexist and the solution converges to periodic orbit or to $\hat{x}^{7}$, respectively (Fig. 2, 3 ). With a denser filter, the smaller algae are filtered out more efficiently; because of the nonlinear effects of algal size on ecological parameters, a broader spectrum of

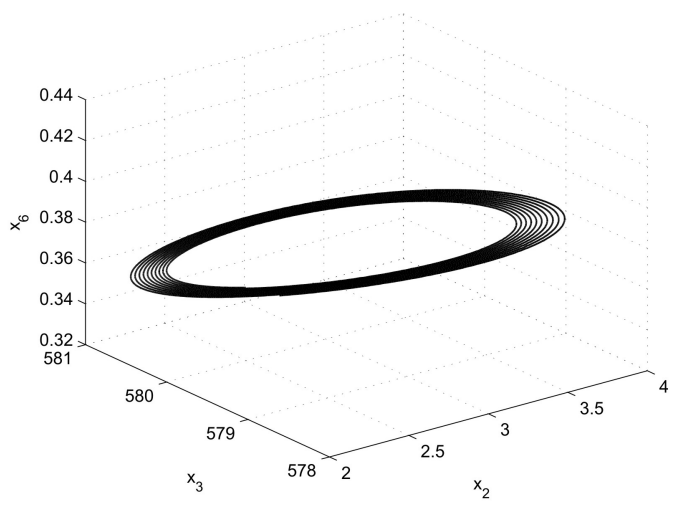

Figure 2: Numerical solution of system (1) for initial condition $x_{1}^{0}=18.2, x_{2}^{0}=0.8, x_{3}^{0}=83.1, x_{4}^{0}=$ $10, x_{5}^{0}=10, x_{6}^{0}=0.2, u=1.5$ and constant environmental condition $(t=120$ in Table 1$)$

species of different sizes is able to survive in the system under the environmental conditions. For the model presented with one growth-limiting nutrient we get that the model exhibits competitive exclusion, only two species of algae are able to survive. Detail analysis of similar systems is given for example in (Kmet et al., 2004) and (Scheffer et al., 2000).

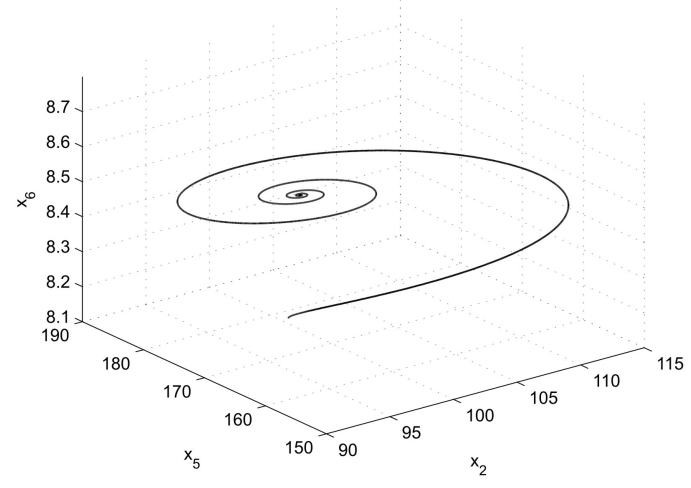

Figure 3: Numerical solution of system (1) for initial condition $x_{1}^{0}=40.2, x_{2}^{0}=98, x_{3}^{0}=0.16, x_{4}^{0}=$ $0.6, x_{5}^{0}=172.9, x_{6}^{0}=8.1, u=10.5$ and constant environmental condition $(t=120$ in Table 1$)$

\section{OPTIMIZATION}

In this section we are interested in the ability of Daphnia to adapt both the filtration area and filter density to the amount and size structure of the food particles (algae) population. We assume that filtration in aquatic filter feeders is an optimal process of maximal feeding strategy. We will investigate two strategies (Kmet et al., 2004):

1) instantaneous maximal biomass production as a goal function (local optimality), i.e., 


$$
\dot{x}_{6}=f_{6}(x, u, t) \rightarrow \max
$$

in respect to $u$ for all $t$,

2) integral maximal biomass (global optimality), i.e.,

$$
J(u)=\int_{0}^{T} x_{6}(t) d t
$$

\section{LOCAL OPTIMALITY}

In the case of strategy 1 , we maximize the following function

$$
J(u)=\sum_{i=2}^{5} \frac{d_{3} a_{9} E_{i}(u) x_{i}}{\left(x_{i}+a_{4}\right)} .
$$

This function attains its maximum on the interval $\left(u_{2}, u_{5}\right)$, where $u_{2}<u_{3}<u_{4}<u_{5}$.

\section{GLOBAL OPTIMALITY}

In case of global optimality we have the following optimal control problem: to find a function $\hat{u}(t)$, for which the goal function $J(\hat{u})=\int_{0}^{T} x_{6}(t) d t$ attains its maximum (i.e. $-\int_{0}^{T} x_{6}(t) d t \rightarrow$ min $)$ under the constraints $c_{1}(x, u)=u_{\text {min }}-u \leq 0, c_{2}(x, u)=u-u_{\max } \leq 0$, where $\mathrm{T}$ denotes the fixed lifetime of an individual Daphnia. We introduce an additional state variable

$$
x_{0}(t)=\int_{0}^{t} x_{6}(s) d s .
$$

We were led to the following optimal control problems: Maximize

$$
x_{0}\left(t_{f}\right)
$$

under the constraints

$$
\begin{aligned}
& c_{1}(x, u)=u_{\min }-u \leq 0 \\
& c_{2}(x, u)=u-u_{\max } \leq 0 .
\end{aligned}
$$

\section{NEURAL NETWORK SOLUTION OF OPTIMAL CONTROL PROBLEM}

Direct optimization methods for solving the optimal control problem are based on a suitable discretization of (4). Choose a natural number $N$ and let $t_{i} \in[0, T], i=$ $0, \ldots, N$, be an equidistant mesh point with $t_{i}=t_{0}+$ $i h, i=1, \ldots, N$, where $h=\frac{t_{f}-t_{0}}{N}$. Let the vectors $x^{i} \in R^{7}, u^{i} \in R, i=1, \ldots, N$, be approximation of state variable and control variable $x\left(t_{i}\right), u\left(t_{i}\right)$, respectively at the mesh point. Discretization of optimal control problem (4) with constraints and state equation (1) using

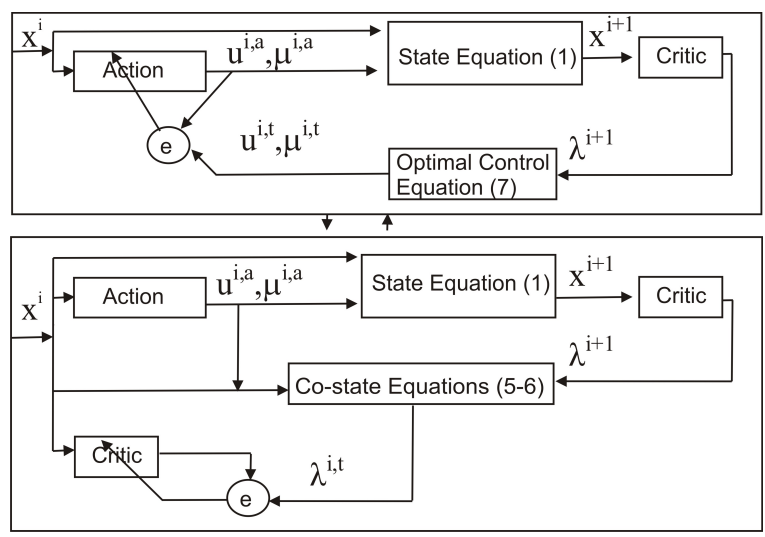

Figure 4: Architecture of adaptive critic feed forward network synthesis, $x^{i}$-input signal to the action and critic network, $u^{i, t}, \mu^{i, t}$ and $\lambda^{i, t}$ output signal from action and critic network, respectively.

Euler's approximation and first order optimality condition of Karush-Kuhn-Tucker (Polak, 1997) leads to the following optimization problem: Minimize

$$
-x_{0}^{N}
$$

subject to

$$
\begin{aligned}
x^{i+1}= & x^{i}+h F\left(x^{i}, u^{i}\right) \\
& i=0, \ldots, N-1, \\
\lambda^{i}= & \lambda^{i+1}+h \lambda^{i+1} F_{x^{i}}\left(x^{i}, u^{i}\right) \\
& +\mu^{i} c_{x^{i}}\left(x^{i}, u^{i}\right), \\
& i=N-1, \ldots, 0, \\
\lambda_{0}^{i}= & -1, i=0, \ldots, N-1 \\
\lambda^{N}= & (-1,0,0,0,0,0,0,0,0,0), \\
0= & h \lambda^{i+1} f_{u^{i}}\left(x^{i}, u^{i}\right) \\
& +\mu^{i} c_{u^{i}}\left(x^{i}, u^{i}\right),
\end{aligned}
$$

where the vector function

$$
F(x, u)=\left(-x_{6}, F_{1}(x, u), \ldots, F_{6}(x, u)\right)
$$

is given by (4) and by right-hand side of (1) and $\lambda$ and $\mu$ are Lagrange multipliers for state and inequality constraints, respectively. To solve optimal control problem, we use adaptive critic and recurrent neural netrork. In the adaptive critic synthesis, the critic and adaptive network were selected such that they consist of six and two subnetworks, respectively, each having 6-18-1 structure (i.e. six neurons in the input layer, eighteen neurons in the hidden layer and one neuron in the output layer). The proposed adaptive critic neural network is able to meet the convergence tolerance values chosen by us, which leads to satisfactory simulation results. For detail explanation of adaptive critic neural network see (Kmet, 2009), (Padhi et al., 2006) and (Padhi et al., 2001). To solve equations (7) we are concerned the following nonlinear projection equation (for detail description see (Xia 
et al., 2007)):

$$
\alpha G\left(P_{X}(u)\right)+u-P_{X}(u)=0,
$$

where $\alpha>0$ is a constant $G: R^{l} \rightarrow R^{l}, X=\{u \in$ $\left.R^{l} \mid d_{i} \leq u_{i} \leq h_{i}\right\}$ and $P_{X}: R^{l} \rightarrow X$ is a projection operator defined by $P_{X}(u)=\left(P_{X}\left(u_{1}\right), \ldots, P_{X}\left(u_{l}\right)\right.$

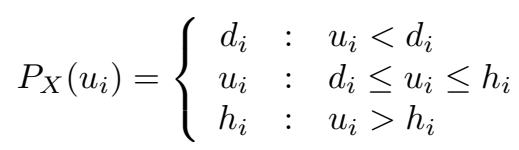

which can be solved by the following dynamic model

$$
\dot{u}(t)=-\beta\left(\alpha F\left(P_{X}(u)\right)+u-P_{X}(u)\right) .
$$

A system described by (9) can be realized by a recurrent neural network with a single-layer structure. The asymptotic and exponencial stability of the present neural network in (9) are proven by (Xia et al., 2007). Equilibrium points of (9) coincide with solutions of (8). Simulations using MATLAB show that proposed neural network is able to solve nonlinear optimal control problem with state and control constraints. Our results are quite similar to those obtained in (Kmet et al., 2004). The results of numerical solutions (Figs. 5 - 6) have shown that optimal strategies $\tilde{u}(t)$ and $\bar{u}(t)$, based on short or longterm perspective respectively, have different time trajectory for different values of $F a z$ (sedimentation function), Temp (water temperature) and $I_{0}$ (light intensity) $(t=120,210)$. When $\bar{u}(t)$ is optimal (what is valid
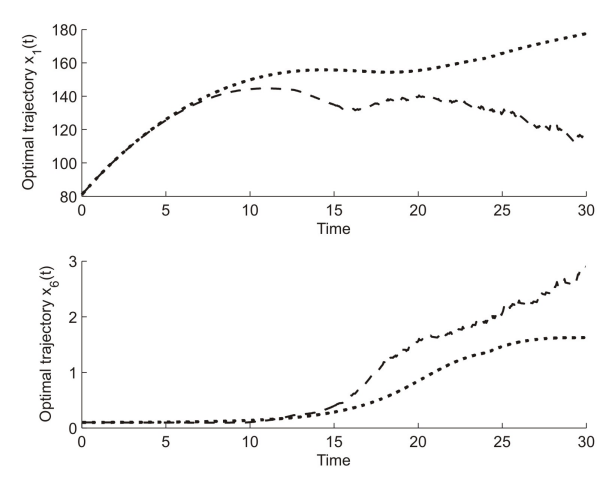

Figure 5: Simulation results - local optimality (dotted line), global optimality (dashed line) for initial condition $x_{1}^{0}=80.4, x_{2}^{0}=0.4, x_{3}^{0}=0.3, x_{4}^{0}=0.2, x_{5}^{0}=$ $0.1, x_{6}^{0}=0.1$ and constant environmental condition $(t=120$ in Table 1$)$

according to numerical results) then $J(\bar{u}(t)) \geq J(\tilde{u}(t))$, i.e., the total biomass for the short-term perspective is smaller or maximally equal to the biomass for the longterm perspective. The numerical results have shown that the initial conditions consider $J(\bar{u}(t))>J(\tilde{u}(t))$ (see Table 4). The higher biomass of zooplankton obtained points in the case of integral formulation towards the assumption that the organisms do better if they do not react only to the immediate changes but also develop mechanisms consistent with more long-term consideration.
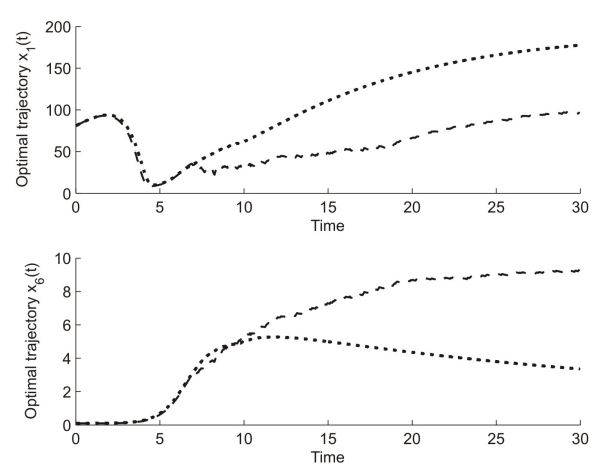

Figure 6: Simulation results - local optimality (dotted line), global optimality (dashed line) for initial condition $x_{1}^{0}=80.4, x_{2}^{0}=0.4, x_{3}^{0}=0.3, x_{4}^{0}=0.2, x_{5}^{0}=$ $0.1, x_{6}^{0}=0.1$ and constant environmental condition ( $t=210$ in Table 1 )

Table 4: Results of goal function evaluations for local and global optimality

\begin{tabular}{c|c|c}
\hline \hline Value of goal function & $\mathrm{t}=120$ & $\mathrm{t}=210$ \\
\hline & & \\
local $J(\tilde{u})$ & 18.4 (Fig.5) & 105.1 (Fig. 5) \\
global $J(\bar{u})$ & 27.9 (Fig. 6) & 178.4 (Fig. 6) \\
\hline \hline
\end{tabular}

Numerical simulations have shown that dynamics of system (1) is different for fixed $u$ and for short-term or long-term strategy, respectively. If we use optimal filtering rate, the results of short-term or long-term optimization all the species $x_{2}, x_{3}, x_{4}, x_{5}$ survival in ecosystem (see Fig. 7, 8).

\section{CONCLUSION}

We considered a simple ecologycal model. One parameter analysis of existence and stability of equilibria was carried out. It is shown that the model has rich dynamics. Also a single network adaptive critic approach is presented for optimal control synthesis with control and state constraints. We have formulated, analysed and solved an optimal control problem related to the optimal uptake of nutrient by Daphnia. Using MATLAB, a simple simulation model based on adaptive critic neural network was constructed. Numerical simulations have shown that the adaptive critic neural network is able to solve nonlinear optimal control problem with control and state constraints and it explains feeding adaptation of filter feeders of Dapnia.

$*$

Acknowledgment. The author is grateful to the referee for his valuable suggestions. The paper was worked out 


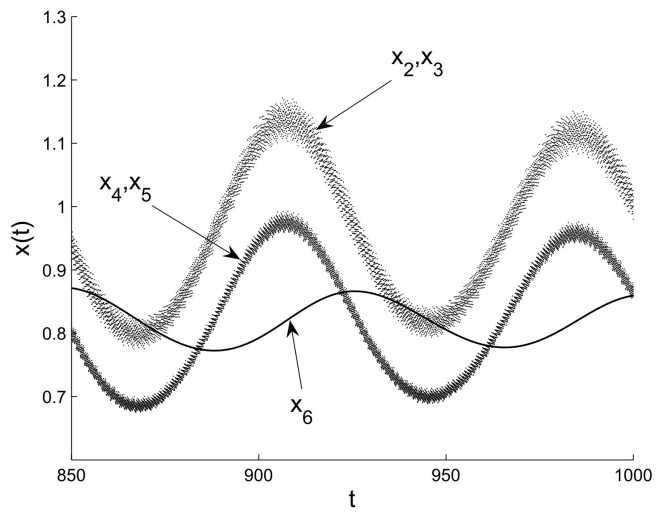

Figure 7: Numerical solution of system (1) for initial condition $x_{1}^{0}=80.3, x_{2}^{0}=0.4, x_{3}^{0}=0.3, x_{4}^{0}=$ $0.2, x_{5}^{0}=0.1, x_{6}^{0}=0.1, u$-local optimality solution and constant environmental condition $(t=120$ in Table 1)

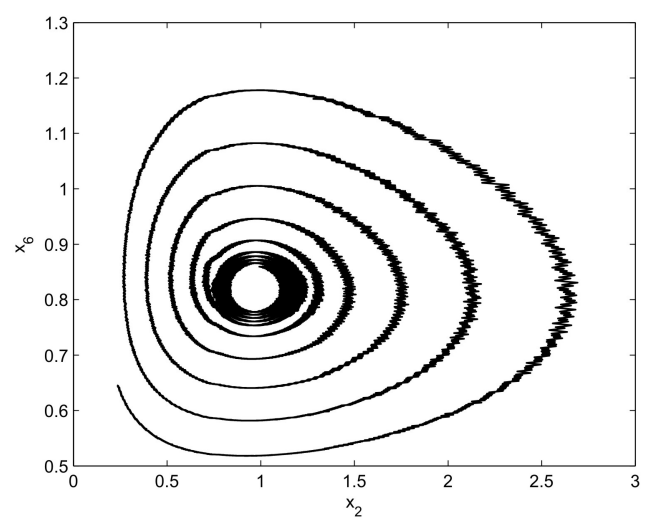

Figure 8: Trajectory of system (1) for initial condition $x_{1}^{0}=80.3, x_{2}^{0}=0.4, x_{3}^{0}=0.3, x_{4}^{0}=0.2, x_{5}^{0}=$ $0.1, x_{6}^{0}=0.1, u$-local optimality solution and constant environmental condition $(t=120$ in Table 1$)$

as a part of the solution of the scientific project number KEGA 004UJS-4/2011.

\section{REFERENCES}

Buskens,Ch. and Maurer, H. (2000). SQP-methods for solving optimal control problems with control and state constraints: adjoint variable, sensitivity analysis and real-time control. Journal of computational and applied mathematics 120:85108.

Hornik,M., Stichcombe, M. and White, H. (1989). Multilayer feed forward networks are universal approximators. Neural Networks 3:256-366.

Hsu, S. B., Hubebell, S., Waltman, P. (1977). A Mathematical Theory for Single-nutrient Competition in Continuous Cultures of Micro-organisms. SIAM J. APPL. MATH. 32:366383.
Kirk, D. E. (1989). Optimal Control Theory: An Introduction. Dover Publications, Inc. Mineola New York.

Kmet, T. (2009) Neural network simulation of nitrogen transformation cycle. In: ECMS 2009 - European Conference on Modelling and Simulation : Madrid, June 9th - 12th, 2009. Madrid: Universidad Rey Juan Carloc . 352-358.

Kmet, T. and Straskraba, M. (2004). Feeding adaptation of filter feeders: Daphnia. Ecological Modelling . 178:313-327.

Padhi, R., Unnikrishnan, N., Wang, X. and Balakrishnan,S. N. (2001). Adaptive-critic based optimal control synthesis for distributed parameter systems. Automatica 37:1223-1234.

Padhi, R., Balakrishnan, S. N. and Randoltph, T. (2006). A single network adaptive critic (SNAC) architecture for optimal control synthesis for a class of nonlinear systems. Neural Networks 19:1648-1660.

Polak, E. (1997) Optimization Algorithms and Consistent Approximation. Springer Verlag, New York, Berlin Heidelberg.

Pontryagin, L. S., Boltyanskii, V. G., Gamkrelidze, R. V. and Mischenko, E. F. (1983). The Mathematical Theory of Optimal Process. Moscow Nauka (in Russian)

Rumelhart, D. F., Hinton, G. E. and Wiliams, R. J. (1987). Learning internal representation by error propagation. In: Rumelhart, D. E., McClelland, D. E., and PDP Research Group. Parallel Distributed Processing Vol 1:Foundation. The MIT Press, Cambrige MA 1:318-362.

Scheffer, M .C.,Rinaldi S., Kuznetsov Y.A. (2000). Effects of fish on plankton dynamics:a theoretical analysis. Can. J. Fish.Aquat. Sci. 57:1208-1219.

Straskraba, M. and Gnauck, P. (1985). Freshwater Ecosystems. Modelling and Simulation. Developments in Environmental Modelling. Elsevier, Amsterdam,

Smith, H. L. and Waltman, P. (1995). The Theory of the Chemostat. Cambride Univ. Press, Cambrige, UK,

Xia, Y. and Feng, G. (2007). A new neural network for solving nonlinear projection equations. Neural Network, 20:577589. 2007.

Yoshizawa, T. (1975). Stability Theory of periodic Solutions and Almost Periodic Solutions. Springer, New York

Zaret, T. M. (1980). Predation and Freshwater Communities. Yale Univ. Press, New Haven,

\section{AUTHOR BIOGRAPHIES}

TIBOR KMET was born in 1956. His email is tkmet@ukf.sk and his personal webpage can be found at http://www.ki.fpv.ukf.sk.

MARIA KMETOVA was born in 1957. Her email is mkmetova@ukf. sk and her personal webpage can be found at http://www.km.fpv.ukf.sk. 